\title{
COSMETIC BLEPHARITIS
}

\author{
Sarah Darbandi, MD
}

Vice President of Bowden Eye \& Associates

Corresponding Author: sdarbandi@bowdeneye.com

Published: April 16, 2020.

These days makeup has evolved into a magical world of instructional YouTube videos, must-have limitededition palettes, and a world of Instagram accounts dedicated to makeup tutorials, reviews, sales, and easily accessible but sometimes harmful tips. Makeup is more than picking up foundation and mascara at the drugstore. These days what simply gets placed on the eyelids to create a basic look involves layers and layers of sometimes not so friendly ingredients, especially for the dry eye patient. I use myself as an example. When I moved from up north to Florida to become a practicing ophthalmologist at Bowden Eye and Associates, I started having dry eye symptoms of eye fatigue and strain. This was made worse by my increased use of computers all of which seem to be located directly below an AC vent, which in Florida is going strong all year round. Eventually, I had the lipiflow procedure and my symptoms resolved. I use oasis plus tears, now the true tear, warm compresses, eyelid scrubs, and I have the lipiflow procedure yearly to make sure my symptoms don't return. At the same time, I continue to hold VIB Rouge status at Sephora and diamond status at Ulta. If you don't know what this means, basically, I buy a lot of makeup. Even when my eyes were most symptomatic from dryness, I continued to wear what I consider the basics ... eyeshadow primer, setting powder, eyeshadow, concealer, eyeliner, mascara primer, mascara, and the occasional glitter glue, or pressed glitter. Our patients are doing the same things, and then some.

For many of the dry eye patients that I see in the clinic, for corneal ulcers or other inflammatory pathologies, and even for postoperative follow up on corneal transplants and cataracts, I will often see the tide line of eyeliners, the remnants of mascara, or the sheen of foundation powders blanketing the corneal surface. These are often patients that state they haven't worn makeup in weeks. Makeup is tenacious. You can pretty much swim in many of the products without it budging. What's most important for us to consider as physicians are not only the carcinogenic chemicals or fragrances that are being placed around a compromised ocular surface, but also how the makeup gets there. The most common offender is eyeliner. It gets placed directly over the "waterline" which refers to the opening of the meibomian glands. The glands then get plastered closed by the thick waxes in eyeliners that keep them from moving throughout 12-15 hours of wear, and in some patients, who aren't diligently removing makeup, while they sleep as well. Sometimes even the makeup removers, many of which contain alcohol-based products or fragrances, are irritating to the eyes, dry the delicate eyelid skin, or cause mechanical irritation of the eyelids and lashes. Most often, particles of makeup get left behind leaving a scaffold to support a biofilm of bacteria, which then 
contributes to inflammation of the ocular surface. And, here we thought makeup was making us look pretty.

Dry eye is a multifactorial disease, and the importance here when discussing makeup is that it gets considered when treating these patients. As a basic regimen, I recommend patients avoid using certain products around the eyes, especially directly over the meibomian glands. I recommend using certain products and brands over others, and I recommend specific makeup removers that are less irritating. Just as you wouldn't let a patient with a contact-lens-associated ulcer continue wearing their contact lenses, we can't let the dry eye patients continue adding waxy, irritating chemicals to their lids daily. Sometimes, we must recommend a break from makeup. Getting even the most severe dry eye patient to leave the house without makeup is a difficult fight that you will have a hard time winning. However, while I find most patients won't give up makeup completely, they will be very receptive to knowing which chemicals to avoid, and which brands to seek out. If we educate ourselves and our patients, we can empower them to make better decisions, that are better for their long-term eye health. Some patients will even appreciate the doctor's orders to head to Sephora to buy better makeup. 